\title{
Seeing more with scanning electron microscopy
}

\section{Richard Young and Laurent Roussel}

An extreme-high-resolution instrument is opening up new possibilities for industrial and research applications, expanding what was previously thought possible.

The scanning electron microscope (SEM) continues to be a key tool in the imaging, metrology, and chemical characterization of nanoscale materials and structures. The SEM offers unique benefits in terms of ease of sample preparation and the ability to image surface details of bulk materials and 3D structures. However, fundamental limitations in beam performance remain, most notably as chromatic aberration effects, which become larger as the beam voltage is reduced below a few kilovolts, restricting achievable resolution.

The introduction of FEI's Magellan ${ }^{\mathrm{TM}}$ extreme-high-resolution (XHR) SEM has shown that subnanometer resolution can be achieved at low beam voltages, revealing ultrafine surface detail. ${ }^{1-3}$ The XHR SEM uses a monochromator to reduce the effects of chromatic aberrations within the electron source, resulting in a more tightly focused electron beam. In addition, we are able to take advantage of the instrument's advances in optics, modularity, platform stability, and cleanliness to explore new avenues, such as high-resolution imaging with beam voltages as low as $50 \mathrm{~V}$ and all the way up to $30 \mathrm{kV}$. For the first time, complementary information from the surface and internal structure at the true nanometer level is obtained in the same SEM.

The importance of improved imaging at low beam voltages is shown by considering polymeric resists used for semiconductor lithography. These resists can be highly sensitive to beam exposure, for example, shrinking when exposed to just one or two scans at a beam landing voltage of $1 \mathrm{kV}$ : see Figure 1(a). With such a sample, operating at a beam landing voltage of $500 \mathrm{~V}$ in the XHR SEM-see Figure 1(b)—produced excellent results with minimal beam effects and good signal-to-noise ratio, a result also observed in other resist materials that we investigated.
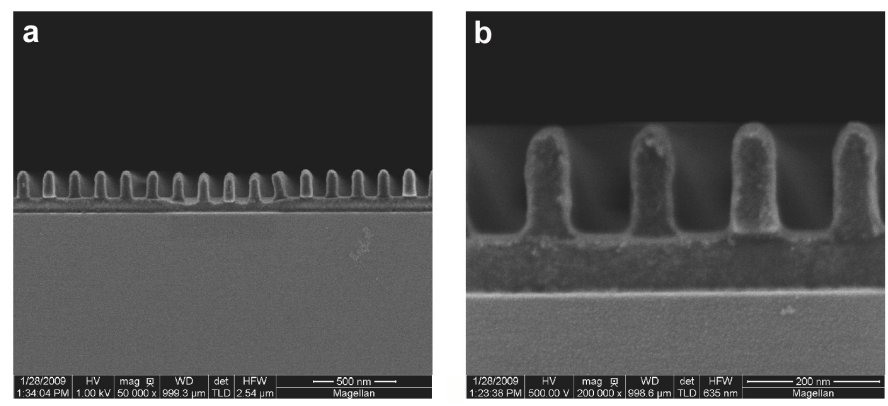

Figure 1. Benefits of low-voltage imaging of semiconductor resist samples (argon fluoride photoresist for 193nm wavelength exposure). At a primary beam voltage of $1 \mathrm{kV}(\mathrm{a})$ the resist starts shrinking and distorting as soon as imaging is started: see the central region where higher magnification imaging was attempted. Reducing the voltage to $500 \mathrm{~V}$ (b) enabled excellent images to be obtained without noticeable beam damage. (Imaged in cooperation with Qimonda, Munich, and I. Gestmann and R. Lehmann, FEI.)

The key technical development of the system is the monochromated beam for XHR imaging. This is provided by an electrostatic Schottky-field emission gun (FEG) module that can also produce large probe currents for analytical applications. The three modes of operation of this module are shown in Figure 2. The extractor contains two apertures, defining an axial and off-axial beam, while a second aperture plane has an axial aperture and a small, off-axis slit that is used to monochromate the off-axis beam. A deflector is then used to direct either the axial or off-axis beam into the SEM column, where a final beam-limiting aperture determines the probe current.

In the first mode of operation, the axial beam is used with gun lens turned off, while in the second the lens is turned on to deliver more beam current. The third mode (called UC mode for UniColore) uses the off-axis monochromated beam. Here, the beam is dispersed due to its off-axis traversal through the strong gun lens, after which a small energy window of the beam is selected by the slit aperture. This design enables the source energy spread to be reduced from a typical value of 0.7 to $0.15 \mathrm{eV}$,

Continued on next page 


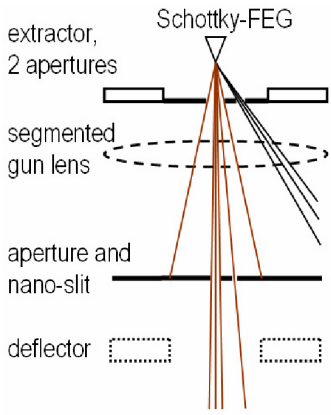

mode 1

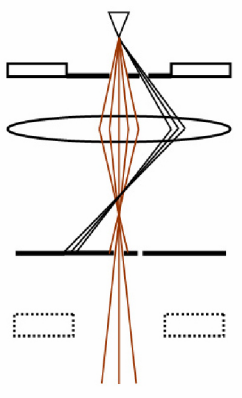

mode 2

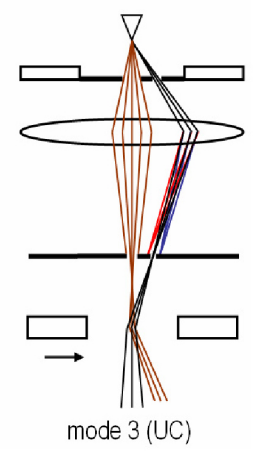

mode 3 (UC)
Figure 2. The three modes of the XHR electron source module: (1) standard-on-axis mode, (2) high-current, and (3) UC (monochromator) mode. Schottky-FEG: Schottky-field emission gun.

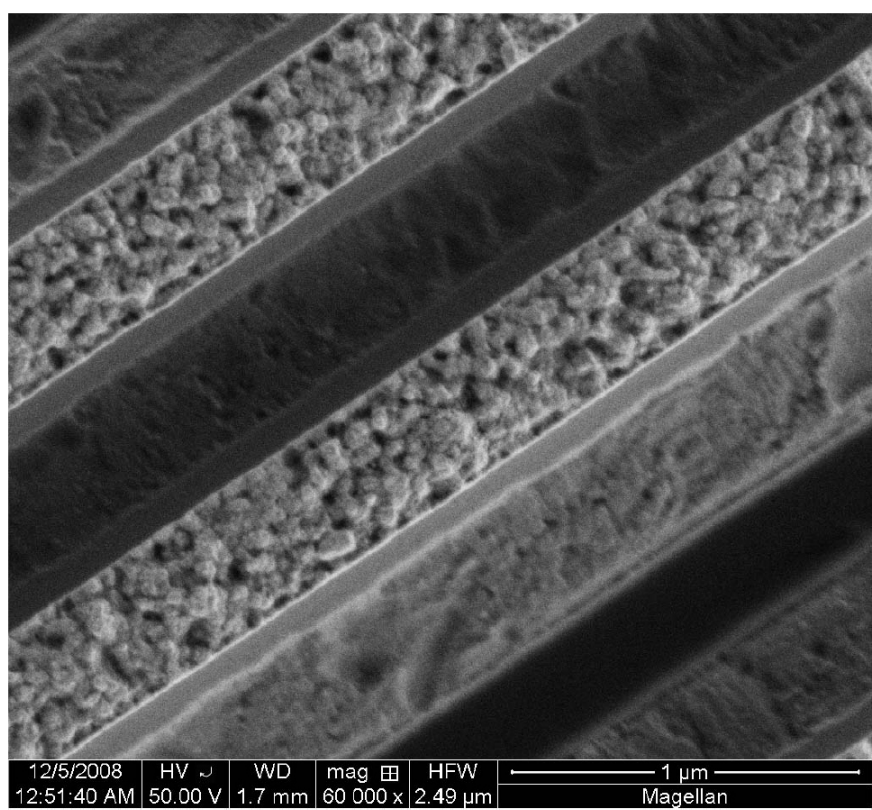

Figure 3. XHR SEM image of a deprocessed semiconductor device image with a beam voltage of $50 \mathrm{~V}$ (combines UC mode with beam deceleration). ${ }^{3}$ (Sample courtesy of STMicroelectronics Malta/Grenoble. Image courtesy of I. Gestmann, FEI.)

producing a dramatic improvement on low-beam-voltage performance (see Figure 3), without compromising system operation in the other modes.

XHR SEM takes today's high-resolution SEM applications and extends them into a new regime of sub-nanometer resolution at low beam voltages. The electron source monochromator used on the Magellan makes a significant improvement in SEM capability, without increasing system complexity or diminishing ease of use. For semiconductor manufacturers, this means continuing to use the SEM for more node shrinks than was previously thought possible. For the researcher, it opens up possibilities for imaging nanoscale structures and accessing greater information from sample surfaces, while maintaining flexibility for operation over the whole beam-voltage range, and for high-resolution analysis and nano-prototyping.

As a next step, we are investigating what benefits these system improvements will bring to the spatial resolution of chemical analysis with the latest generation of x-ray detectors. In addition, FEI is focusing on exploring how the resolution improvements in XHR imaging can extend SEM into applications that normally require the higher-resolution capabilities of transmission electron microscopy.

The authors are indebted to the entire FEI Magellan development team, and also gratefully acknowledge the support of the Smart Mix Programme of the Netherlands Ministry of Economic Affairs and the Netherlands Ministry of Education, Culture, and Science.

\section{Author Information}

\section{Richard Young}

FEI Company

Hillsboro, OR

Richard Young is a technologist within the Nano Electronics Division at FEI.

\section{Laurent Roussel}

FEI Company

Eindhoven, The Netherlands

Laurent Roussel is marketing manager within the Nano Research Division at FEI.

\section{References}

1. R. Young, T. Templeton, L. Roussel, I. Gestmann, G. van Veen, T. Dingle, and S. Henstra, Extreme high resolution SEM: a paradigm shift, Microsc. Today 16 (4), pp. 24-28, 2008.

2. R. Young, S. Henstra, J. Chmelik, T. Dingle, A. Mangnus, G. van Veen, and I. Gestmann, XHR SEM: enabling extreme high resolution scanning electron microscopy, Proc. SPIE 7378, p. 737803, 2009. doi:10.1117/12.824749

3. L. Y. Roussel, D. J. Stokes, I. Gestmann, M. Darus, and R. J. Young, Extreme high resolution scanning electron microscopy (XHR SEM) and beyond, Proc. SPIE 7378, p. $73780 \mathrm{~W}, 2009$. doi: $10.1117 / 12.821826$ 